\title{
Editorial
}

\section{Special Issue on Advanced Learning Methods for Complex Data}

\author{
Maurizio Atzori *(D) and Barbara Pes *(D) \\ Dipartimento di Matematica e Informatica, Università degli Studi di Cagliari, Via Ospedale 72, \\ 09124 Cagliari, Italy \\ * Correspondence: atzori@unica.it (M.A.); pes@unica.it (B.P.)
}

Received: 22 December 2018; Accepted: 24 December 2018; Published: 27 December 2018

check for updates

\begin{abstract}
The importance of data mining methods has increased dramatically in recent years, making this research area relevant and challenging to extract actionable knowledge from complex data. Indeed, new algorithms and machine learning methods are constantly being explored to deal with domains that present multiple challenges including high-dimensionality, heterogeneity of features, and complex relationships between data objects. This special issue aims at discussing emerging approaches for learning from complex data, including text data, images, and social media data.
\end{abstract}

Keywords: data mining and knowledge discovery; artificial intelligence; machine learning; complex data

Though the crucial role of data mining for knowledge discovery has long been recognized [1], this research area is now experiencing a new and explosive growth, due to the exponential increase of the amount of data, as well as to the increasing complexity of these data. In almost all the application domains, indeed, the variety of relationships among the data objects, besides the number and heterogeneity of the objects' features, makes imperative the adoption of innovative approaches for exploring data and learning their underlying patterns.

Further, in the era of big data and internet of things, additional challenges arise from the multiple modalities, multiple resources, and multiple formats through which the information is made available, giving rise to the need for more flexible paradigms for both data modelling and data analysis $[2,3]$. In this perspective, the data mining and machine learning communities are increasingly leveraging multiple contributions from a variety of fields, including semantic web and cloud technologies [4-6].

A number of proposals from this fast-evolving research area are discussed in our special issue on "Advanced Learning Methods for Complex Data", with a special focus on approaches that exploit semantic information when dealing with complex data and learning from them.

Among the submissions received, all of which went through a rigorous peer-review process, three papers have been selected for publication. In particular, two of them are extended versions of previous research presented at the Data Exploration in the Web 3.0 Age (DEW) track of the IEEE WETICE conference [7,8]. A brief summary of the works included in the special issue is provided in what follows.

The first paper "Aspect Term Extraction Based on MFE-CRF", by Yanmin Xiang, Hongye He and Jin Zheng [9] focuses on aspect term extraction in aspect-based sentiment analysis, which is one of the hot spots in natural language processing. They propose multi-feature embedding (MFE) clustering based on the conditional random field (CRF) model to improve the effect of aspect term extraction in aspect-based sentiment analysis, showing that MFE can improve both the precision and recall rates of the CRF model. 
The second paper "Integration of Context Information through Probabilistic Ontological Knowledge into Image Classification", by Andrea Apicella, Anna Corazza, Francesco Isgrò and Giuseppe Vettigli [10], proposes an innovative combination where a classic classifier for object recognition on images is enhanced by integrating knowledge from a probabilistic ontology. This hybrid approach with contextual information can show relations that exist between the different segments, useful in many applications, and also leads to better classification accuracy.

Finally, the third paper "Evaluating User Behaviour in a Cooperative Environment", by Enrico Bazzi, Nunziato Cassavia, Davide Chiggiato, Elio Masciari, Domenico Saccà, Alessandra Spada, and Irina Trubitsyna [11], addresses the problem of understanding the influence of user behaviours such as searches and suggestions across a social network. Network dynamics are measured using three different metrics, and a number of techniques are used to set up an experimental evaluation that empirically shows how users influence each other in a cooperative environment when a kind of reward is provided.

Acknowledgments: The guest editors would like to thank the authors for their valuable submissions, the MDPI editors for their constant support in the special issue production, and the reviewers for their precious and constructive comments.

Conflicts of Interest: The guest editors declare no conflict of interest.

\section{References}

1. Fayyad, U.M.; Piatetsky-Shapiro, G.; Smyth, P. From data mining to knowledge discovery: An overview. In Advances in Knowledge Discovery and Data Mining; American Association for Artificial Intelligence: Menlo Park, CA, USA, 1996; pp. 1-34.

2. Wu, X.; Zhu, X.; Wu, G.Q.; Ding, W. Data mining with big data. IEEE Trans. Knowl. Data Eng. 2014, 26, 97-107.

3. Tsai, C.W.; Lai, C.F.; Chiang, M.C.; Yang, L.T. Data Mining for Internet of Things: A Survey. IEEE Commun. Surv. Tutorials 2014, 16, 77-97. [CrossRef]

4. Argiolas, M.; Atzori, M.; Dessì, N.; Pes, B. Dataspaces Enhancing Decision Support Systems in Clouds. Int. J. Web Portals 2012, 4, 35-55. [CrossRef]

5. Ristoski, P.; Paulheim, H. Semantic Web in data mining and knowledge discovery: A comprehensive survey. J. Web Semant. 2016, 36, 1-22. [CrossRef]

6. Dou, D.; Wang, H.; Liu, H. Semantic data mining: A survey of ontology-based approaches. In Proceedings of the 2015 IEEE International Conference on Semantic Computing (ICSC), Anaheim, CA, USA, 7-9 February 2015; pp. 244-251.

7. Atzori, M.; Dessì, N.; Pes, B. Track Summary Report for the Data Exploration in the Web 3.0 Age (DEW) Track. In Proceedings of the 26th IEEE International Conference on Enabling Technologies: Infrastructure for Collaborative Enterprises (WETICE), Poznan, Poland, 21-23 June 2017; pp. 155-157.

8. Atzori, M.; Pes, B. Summary Report for the Data Exploration in the Web 3.0 Age (DEW) Track. In Proceedings of the 27th IEEE International Conference on Enabling Technologies: Infrastructure for Collaborative Enterprises (WETICE), Paris, France, 27-29 June 2018; pp. 139-141.

9. Xiang, Y.; He, H.; Zheng, J. Aspect Term Extraction Based on MFE-CRF. Information 2018, 9, 198. [CrossRef]

10. Apicella, A.; Corazza, A.; Isgrò, F.; Vettigli, G. Integration of Context Information through Probabilistic Ontological Knowledge into Image Classification. Information 2018, 9, 252. [CrossRef]

11. Bazzi, E.; Cassavia, N.; Chiggiato, D.; Masciari, E.; Saccà, D.; Spada, A.; Trubitsyna, I. Evaluating User Behaviour in a Cooperative Environment. Information 2018, 9, 303. [CrossRef]

(C) 2018 by the authors. Licensee MDPI, Basel, Switzerland. This article is an open access article distributed under the terms and conditions of the Creative Commons Attribution (CC BY) license (http://creativecommons.org/licenses/by/4.0/). 